OPEN ACCESS

Edited by:

Haoyu Chen,

The Chinese University of Hong

Kong, China

Reviewed by:

Annette Hoskin,

The University of Sydney, Australia

Alessandro Meduri,

University of Messina, Italy

*Correspondence:

Hongling Chen

389221922@qq.com

Specialty section: This article was submitted to

Ophthalmology,

a section of the journal

Frontiers in Medicine

Received: 08 August 2021 Accepted: 04 October 2021

Published: 27 October 2021

Citation:

Chen H, Han J, Zhang X and Jin X

(2021) Clinical Analysis of Adult

Severe Open-Globe Injuries in Central

China. Front. Med. 8:755158

doi: 10.3389/fmed.2021.755158

\section{Clinical Analysis of Adult Severe Open-Globe Injuries in Central China}

\author{
Hongling Chen ${ }^{1 *}$, Junjun Han ${ }^{1}$, Xianliang Zhang ${ }^{1}$ and Xuemin Jin $^{2}$ \\ ${ }^{1}$ Department of Ophthalmology, Henan Eye Institute, Henan Eye Hospital, Henan Provincial People's Hospital, People's \\ Hospital of Zhengzhou University, Zhengzhou, China, ${ }^{2}$ Department of Ophthalmology, The First Affiliated Hospital of \\ Zhengzhou University, Zhengzhou, China
}

Purpose: To describe the characteristics, management, and outcomes of adult severe open-globe injured (OGl) eyes.

Methods: Retrospective chart review of inpatients with initial visual acuity (VA) of light perception (LP) or no light perception (NLP) associated with OGl between 2017 and 2020 at Department of Ophthalmology, Henan Eye Institute, Henan Eye Hospital, Henan provincial People's Hospital.

Results: Six hundred twenty-five eyes of 622 adult patients with initial VA of LP or NLP associated with open-globe injuries (OGIs) were included. The mean age was 47.8 \pm 14.1 years with the range from 18 to 91 years. Significant male predominance was noted (81.5\%). The most common type of these severe OGls was rupture (65.8\%). Traffic accidents accounted for $13.5 \%$ followed by fall/tumble (10.9\%) and nail/wire (10.9\%) of all the severe OGls. Almost half of the injuries happened at workplace (47.2\%). Initially, $78.7 \%$ eyes just received primary debridement and wound closure, while $8.5 \%$ eyes with no possible of anatomical reconstruction received evisceration. After initial management, 350 eyes received subsequent operation, including 239 eyes underwent vitrectomy + silicone oil/(+cataract remove). Finally, over 6 months follow-up, 137 eyes (21.9\%) were eviscerated, 150 eyes (24.0\%) got atrophied, while 132 eyes $(21.1 \%)$ retain some VA. Fifty-three eyes (8.5\%) got VA of 0.3-1.5.

Conclusion: Severe OGls are most seen in the young, middle-aged, and male working population and remain a serious public health problem, resulting in significant vision loss or Evisceration of eyes. Effective preventive measures should be taken for the individuals in these groups.

Keywords: adult, central China, open-globe injuries, trauma, epidemiology

\section{INTRODUCTION}

Open-globe injuries (OGIs) include a full-thickness break or rupture of the cornea and/or the sclera. OGIs are the major cause of unilateral visual loss. Severe OGIs (initial VA of LP or NLP) not only lead to vision loss but also can cause eye loss. OGIs seem more common in developing countries than in developed countries (1). Henan province is a large agricultural province. It is located in central China with rich labor resources. Latest population census shows that the population of Henan was about one hundred million (99,366 thousands). Henan Eye Hospital is 
the 1st or the second largest ophthalmic center in Henan Province and received quite a lot of ocular trauma patients every year in Henan Province.
As we know, OGIs are most seen in working population and remain a serious public health problem, resulting in significant vision loss. In severe OGIs, eyes had been destroyed or cannot

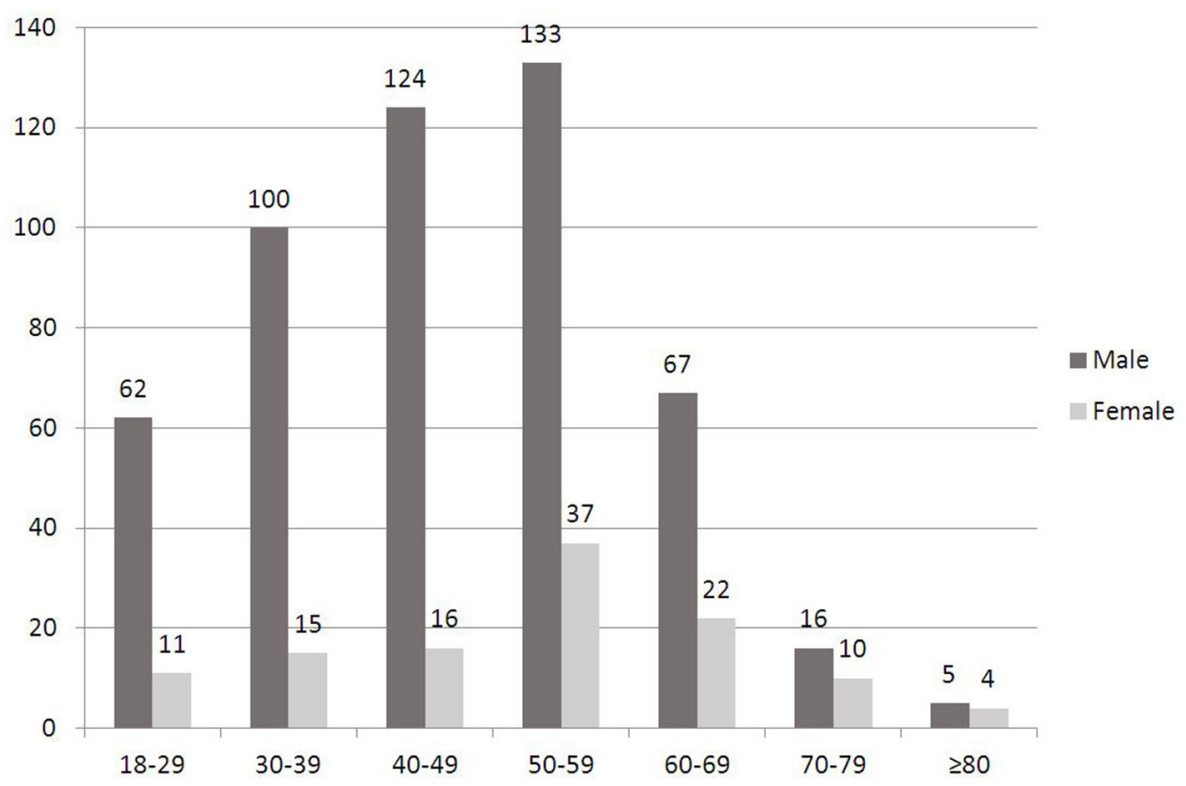

FIGURE 1 | Distribution of gender and age groups of severe adult opened globe injury patients.

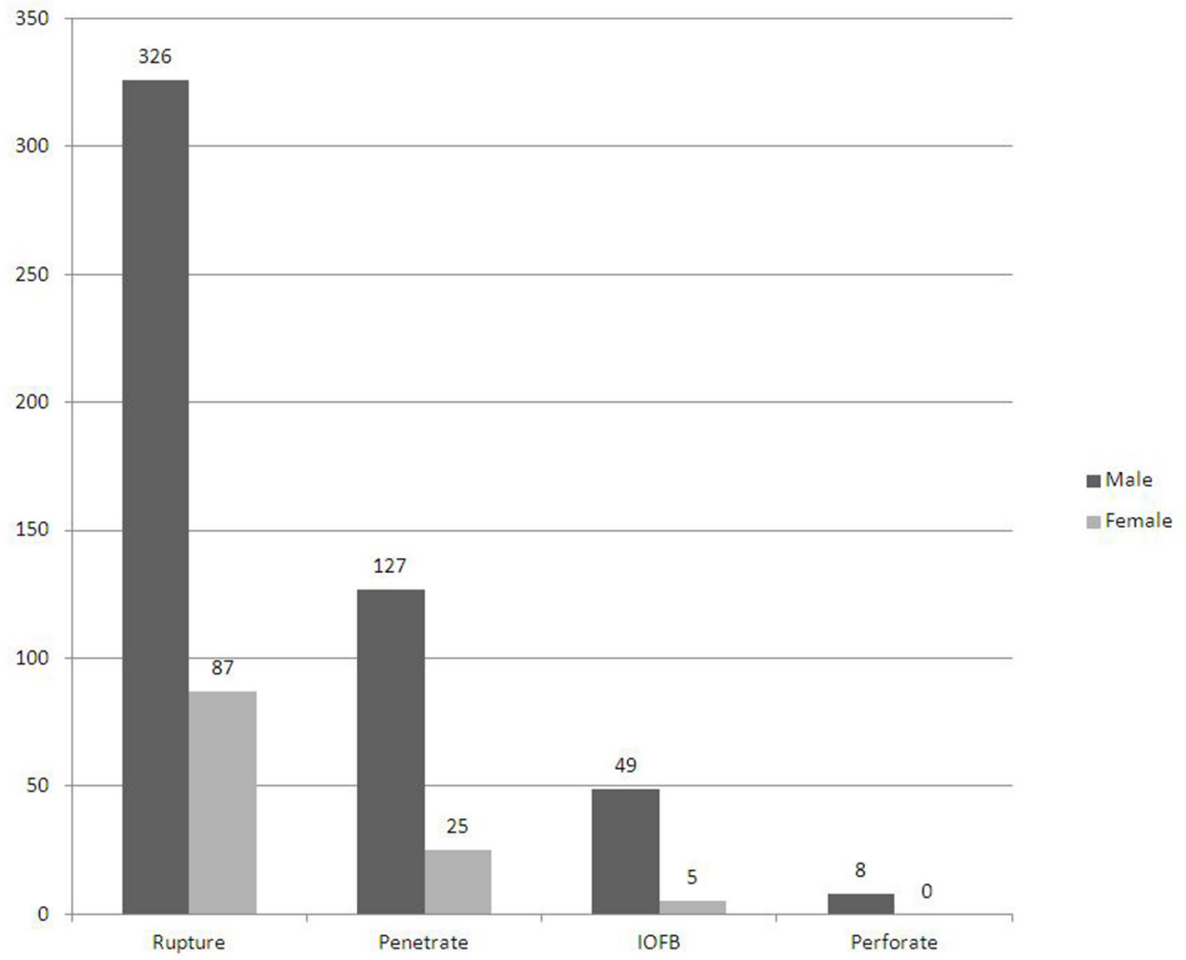

FIGURE 2 | Distribution of gender and types of opened globe injury patients. 
be reconstructed. A good understanding of the characteristics of severe OGIs in adults is required for the determination of preventive measures. In this study, we aimed to separately analyze the characteristics of adult severe OGIs diagnosed and treated at Department of Ophthalmology, Henan Eye Institute, Henan Eye Hospital, Henan provincial People's Hospital.

\section{METHODS}

We retrospectively chart reviewed all the patients diagnosed and treated at Department of Ophthalmology, Henan Eye Institute, Henan Eye Hospital, Henan provincial People's Hospital between 2017 and 2020. The OGI patients aged over 18 and with initial VA of LP or NLP were included into the study. Age, gender, mechanism of injury, surgical options, and outcomes were analyzed. Ethics approval for the study was granted by Henan Eye Institute, Henan Eye Hospital, Henan provincial People's Hospital Human Research Ethics Committee. The study adhered to the tenets of the Declaration of Helsinki.

\section{Classification}

Open-globe injuries were classified according to the Birmingham Eye Trauma Terminology System (2).

The patients were classified into 7 age groups: $18-29,30-39$, $40-49,50-59,60-69,70-79, \geq 80$ years.

\section{Statistical Analysis}

Data were analyzed using Microsoft Office Excel 2007. Continuous and categorical variables were displayed as means \pm standard deviation (SD) and percentages, respectively.

The ethics number is HNEECKY-2021(33).

\section{RESULTS}

In the 4 years, 1,902 cases (1,908 eyes) of OGI patients (including 1,483 eyes of 1,478 patients older than 17 years) were diagnosed and treated at Department of Ophthalmology, Henan Eye Institute, Henan Eye Hospital, Henan provincial People's Hospital. Of all the OGIs, 622 cases (625 eyes) of adult severe OGIs with initial VA of LP or NLP were included in this study. The mean age at the time of injury was $47.8 \pm 14.1$ years with the range from 18 to 91 years. Significant male predominance was noted $(81.5 \%)$. The highest incidence of severe adult OGIs was found in the middle-aged group (50-59, 40-49, 30-39) in male patients (Figure 1). The highest incidence of severe adult OGIs was found in 50-59 age group in female patients. Of the 622 patients, 3 cases had bilateral eye injury. In patients with unilateral eye injury, 299 (48.2\%) eyes were right eye and 320 (51.2\%) were left eye.

In the present study, the most common type of severe OGIs was rupture $(65.8 \%)$ no matter in male (326 eyes) or female (87 eyes) (Figure 2). Traffic accidents accounted for $13.5 \%$ followed by fall/tumble (10.9\%) and nail/wire (10.9\%) of all the severe OGIs (Table 1). Almost half of the injuries happened at workplace $(47.2 \%)$.

Initially, 492 eyes $(78.7 \%)$ received primary debridement and wound closure. Fifty three eyes $(8.5 \%)$ with no possible of
TABLE 1 | Causes of severe open globe injuries.

\begin{tabular}{lcc}
\hline Cause & $\boldsymbol{n}$ & $\%$ \\
\hline Traffic accident & 84 & 13.5 \\
Fall, tumble & 68 & 10.9 \\
Nail, wire & 68 & 10.9 \\
Wood, branch, bamboo & 58 & 9.3 \\
Fireworks, firecrackers & 43 & 6.9 \\
Emery cutter, grinding wheel, electric saw & 37 & 6 \\
Violence & 34 & 5.5 \\
Metal bar & 30 & 4.8 \\
Metal fragments caused by hammering & 23 & 3.7 \\
Metal block, sheet metal & 21 & 3.4 \\
Scissors, knife & 12 & 1.9 \\
Flying stone & 12 & 1.9 \\
Lighter, bottle, bulb explosion & 7 & 1.1 \\
Glass & 5 & 0.8 \\
Falling objects & 4 & 0.6 \\
Metal hook & 3 & 0.5 \\
Toy bullet & 3 & 0.5 \\
Children head & 2 & 0.3 \\
Battery explosion & 2 & 0.3 \\
Flying object from grass trimmer & 1 & 0.2 \\
Water pump explosion & 1 & 0.2 \\
Tire explosion & 1 & 0.2 \\
Pressure cooker explosion & 1 & 0.2 \\
Gas tank explosion & 1 & 0.2 \\
Cock peck & 1 & 0.2 \\
Horn & 1 & 0.2 \\
Others and unknown & 100 \\
Total & 1 & \\
\hline & & \\
\hline
\end{tabular}

TABLE 2 | Primary management.

\begin{tabular}{lcc}
\hline Surgical options & $\boldsymbol{n}$ & $\%$ \\
\hline Wound closure & 492 & 78.7 \\
Evisceration & 53 & 8.5 \\
Comprehensive management & 46 & 7.4 \\
Wound closure + cataract removal & 23 & 3.7 \\
No surgery & 7 & 1.1 \\
Other surgeries & 4 & 0.6 \\
Total & 625 & 100 \\
\hline
\end{tabular}

anatomical reconstruction received evisceration (Table 2). After initial management, 350 eyes received subsequent operation, including 239 eyes underwent vitrectomy + silicone oil/ (+cataract remove) and 58 eyes got eviscerated (Table 3 ). Over 6 months of follow-up, 137 eyes (21.9\%) were eviscerated, 150 eyes $(24.0 \%)$ got atrophied, and 132 eyes $(21.1 \%)$ retain some VA. At the end of follow up, fifty-three eyes (8.5\%) got VA of $0.3-$ 1.5 (Table 4). Visual recovery was much better in eyes with initial VA of LP than that in eyes with initial VA of NLP. In eyes with initial VA of NLP, final vision improved to light perception/ hand 
TABLE 3 | Secondary intervention.

\begin{tabular}{lcc}
\hline Surgical options & $\boldsymbol{n}$ & $\%$ \\
\hline Vitrectomy + silicone oil/(+cataract remove) & 239 & 68.3 \\
Evisceration & 58 & 16.6 \\
Vitrectomy/(+cataract remove) & 38 & 10.8 \\
cataract remove/(+IOL implant) & 15 & 4.3 \\
Total & 350 & 100 \\
\hline
\end{tabular}

TABLE 4 | Final outcomes of 625 eyes with severe OGls.

\begin{tabular}{lccccc}
\hline & \multicolumn{2}{c}{ LP } & & \multicolumn{2}{c}{ NLP } \\
\cline { 2 - 3 } \cline { 5 - 6 } Outcomes & $n$ & $\%$ & & $n$ & $\%$ \\
\hline Loss of follow-up & 42 & 15.4 & & 32 & 9.1 \\
Evisceration & 13 & 4.8 & & 124 & 35.2 \\
Atrophy & 17 & 6.2 & 62 & 17.6 \\
Atrophy with silicone oil & 23 & 8.4 & & 48 & 13.6 \\
Silicone oil dependence & 31 & 11.4 & 49 & 13.9 \\
NLP-CF & 40 & 14.7 & 12 & 3.4 \\
0.01-0.25 & 60 & 22 & 19 & 5.4 \\
0.3-1.5 & 47 & 17.1 & 6 & 1.8 \\
Total & 273 & & 352 & \\
\hline
\end{tabular}

TABLE 5 | Surgery time of 625 eyes with severe OGls.

\begin{tabular}{lcc}
\hline Surgery time & $\boldsymbol{n}$ & $\%$ \\
\hline 0 & 7 & 1.1 \\
1 & 216 & 34.6 \\
2 & 307 & 49.1 \\
3 & 80 & 12.8 \\
4 & 13 & 2.1 \\
5 & 2 & 0.3 \\
Total & 625 & 100 \\
\hline
\end{tabular}

movement in 6 eyes (1.7\%), counting fingers in 5 eyes $(1.4 \%)$, $0.02-0.8$ in 25 eyes $(7.1 \%)$.

Of all the 625 severe OGIs, almost half eyes (49.1\%) received 2 times of surgery (Table 5). The average was 1.8 times. Seven eyes were followed up with medical treatment alone and 2 eyes received 5 times surgeries. One eye that underwent 5 times surgeries achieved VA of 0.1 . Another eye that underwent 5 times surgeries achieved VA of HM.

Of all the 625 severe OGIs, 53 eyes (8.5\%) underwent intraocular lens (IOL) related surgery at last. Thirty-seven eyes underwent IOL implant and 16 eyes underwent sclera suture or intrascleral fixation (3).

Of all the eviscerated 137 eyes, 53 eyes (38.4\%) were eviscerated at the primary surgery, 68 eyes (49.6\%) were eviscerated at the second surgery, 13 eyes $(9.5 \%)$ were eviscerated at the third surgery and 3 eyes $(2.2 \%)$ were eviscerated at the fourth surgery.
Of all the 622 cases of severe OGIs, 2 patients were diagnosed sympathetic ophthalmia. One patient was a 50 year-old male patient who sustained an injury to his right eye when riding a bicycle. Ten weeks later he was diagnosed sympathetic ophthalmia. Another patient was a 41 years old male patient who got his right eye hurt by finger and 8 weeks later he was diagnosed sympathetic ophthalmia.

\section{DISCUSSION}

Open globe injury (OGI) remains a significant public health problem both in developed and developing countries due to their outcomes. This type of injury is more commonly seen in underdeveloped and developing countries than in developed countries (1). It is a major and preventable cause of unilateral visual loss.

In spite of medical and technical advancements, severe OGIs result in substantial visual morbidity and lifelong sequelae. Severe OGIs also impose financial burdens on society, company and patients. According to our study, each severe OGI eye need 1.8 times of surgery. Currently, the average medical fee for each severe OGI eye is about 25,000 RMB in Henan Province. In underdeveloped and developing countries, severe OGIs mean disasters to the patients and their families for each patient is the backbone of the family. In our study, the highest incidence of severe adult OGIs was found in the middle-aged group (30-59). The mean age at the time of injury was $47.8 \pm 14.1$ years.

It seems that patients were younger than those in the study of Fujikawa et al. (4). The mean age of their study populations was $56.7 \pm 21.8$ years in the LP group and $62.3 \pm 21.7$ years in the NLP group. This may indirectly reflect the different condition of aging of population of the society. Another interesting difference between our study and Fujikawa et al. (4) is the male to female ratio. In our study, male to female ratio is $81.5 \%$ while in the study of Fujikawa et al. (4) it is $66.1 \%$. In other studies, male to female ratio was $73.3-80 \%(1,4-12)$. The highest ratio was found in the study of Supreeyathitikul et al. (13) (88.7\%).

In the current study, almost half of the injuries happened at workplace $(47.2 \%)$ which is similar to the study of Supreeyathitikul et al. (13) (45.8\%). In our study, all the severe OGIs cases related to non-wearing of eye protective device. If the patients wear eye protective device, maybe they would not get eyes injured. According to article 54 of $<<$ Safe Production Law of the People's Republic of China $>>$, the departments in charge of supervising and administering production safety, must strictly check, test or accept the matters concerning production safety, which call for examination and approval, in accordance with the relevant laws and regulations and in conformity with the safety conditions and procedures as required by national standards or trade standards. In fact, Safe Production Law is implemented well in formal company. Safe Production Law seems difficult to enforce when employees work for small unformal company or individuals. Legislation needs improvement to prevent ocular trauma and other traumas.

Of all the 625 severe OGIs, 53 eyes (8.5\%) underwent intraocular lens (IOL) related surgery at last. Thirty-seven eyes 
underwent IOL implant and 16 eyes underwent sclera suture or intrascleral fixation (3). For eyes with combined lens capsular and iris deficiency, glued aniridia IOL and glued IOL with iridoplasty maybe the optimal options (14). Unfortunately, they are unavailable for us.

In our study, 53 eyes were eviscerated at the primary management and 58 eyes were eviscerated at the second intervention. Over 6 months of follow-up, a total of 137 eyes underwent evisceration. The rate of evisceration for severe OGIs in all adult OGIs was $9.2 \%(137 / 1,483)$. It is similar to that of other large series $(12,15,16)$. In our study, 124 eyes $(35.2 \%)$ with initial VA of NLP underwent evisceration. The rate is much lower than that in another small series study (17) in which 21 eyes (84\%) were eviscerated in 25 eyes presenting with no light perception (NLP) after open globe injury (OGI). No eyes underwent enucleation in the present study. Zigiotti et al. (18) described a modified standard enucleation.

Before deciding on evisceration/enucleation in severe OGI eyes, reversible causes of vision loss should be excluded including psychological factors (19). Even in situations in which evisceration/enucleation seems inevitable; the ophthalmologist should discuss the possible options with the patient before making a final decision. Primary evisceration/enucleation for severe OGI eyes with NLP in view of risk of sympathetic ophthalmia was a controversial approach. Sympathetic ophthalmia with potential for bilateral blindness is a relative indication for evisceration/enucleation of an injured eye. The use of modern immunosuppressives has also improved treatment and control of sympathetic ophthalmia. In the current study, 2 patients were diagnosed sympathetic ophthalmia after about 2 months later of injury. In consideration of 132 eyes (21.1\%) retain some VA and 53 eyes $(8.5 \%)$ got VA of $0.3-1.5$, primary surgical repair should not be

\section{REFERENCES}

1. Batur M, Seven E, Esmer O, Akaltun MN, Yasar T, Cinal A. Epidemiology of adult open globe injury. J Craniofac Surg. (2016) 27:1636-41 doi: 10.1097/SCS.0000000000003001

2. Kuhn F, Morris R, Witherspoon CD, Mester V. The Birmingham eye trauma terminology system (BETT). J Fr Ophtalmol. (2004) 27:20610. doi: 10.1016/S0181-5512(04)96122-0

3. Postorino M, Meduri A, Inferrera L, Tumminello G, Rechichi M, Caparello $\mathrm{O}$, et al. Scleral pockets for an innovative technique of intrascleral fixation of intraocular lens. Eur J Ophthalmol. (2020) 30:98590. doi: 10.1177/1120672119866018

4. Fujikawa A, Mohamed YH, Kinoshita H, Matsumoto M, Uematsu M, Tsuiki E, et al. Visual outcomes and prognostic factors in open-globe injuries. BMC Ophthalmol. (2018) 18:138. doi: 10.1186/s12886-018-0804-4

5. Mir TA, Canner JK, Zafar S, Srikumaran D, Friedman DS, Woreta FA. Characteristics of open globe injuries in the United States from 2006 to 2014. JAMA Ophthalmology. (2020) 138:268-75. doi: 10.1001/jamaophthalmol.2019.5823

6. Ji YR, Zhu DQ, Zhou HF, Fan XQ. Epidemiologic characteristics and outcomes of open globe injury in Shanghai. Int J Ophthalmol. (2017) 10:1295300. doi: 10.18240/ijo.2017.08.18

7. Li EY, Chan TC, Liu AT, Yuen HK. Epidemiology of openglobe injuries in Hong Kong. Asia Pac J Ophthalmol. (2017) 6:54-8. doi: 10.1097/APO.0000000000000211 abandoned for the risk of sympathetic ophthalmia in eyes with NLP.

In conclusion, this study demonstrated the characteristic, managements, and outcomes of severe OGIs. Severe OGIs are most seen in the young, middle-aged, and male working population and remain a serious public health problem, resulting in significant vision loss or evisceration/enucleation of eyes. Effective preventive measures should be taken for the individuals in these groups. Employers and employees need to be educated on the importance of eye protection. Legislation needs improvement to prevent ocular trauma and other traumas. Finally, we must expand outreach and education to at-risk populations.

\section{DATA AVAILABILITY STATEMENT}

The original contributions presented in the study are included in the article/supplementary material, further inquiries can be directed to the corresponding author.

\section{ETHICS STATEMENT}

The studies involving human participants were reviewed and approved by Ethics Committee of Henan Eye Hospital. The patients/participants provided their written informed consent to participate in this study.

\section{AUTHOR CONTRIBUTIONS}

$\mathrm{HC}, \mathrm{JH}$, and $\mathrm{XZ}$ organized the database. HC performed the statistical analysis and wrote the first draft of the manuscript. All authors contributed to conception and design of the study, manuscript revision, read, and approved the submitted version.

8. Makhrash MA, Gosadi IM. Open globe eye injury characteristics and prognostic factors in Jazan, Saudi Arabia. Saudi Med J. (2016) 37:132833. doi: 10.15537/smj.2016.12.15545

9. Atik SS, Ugurlu S, Egrilmez ED. Open globe injury: demographic and clinical features. J Craniofac Surg. (2018) 29:62831. doi: 10.1097/SCS.0000000000004156

10. Madhusudhan AP, Evelyn-Tai LM, Zamri N, Adil H, Wan-Hazabbah WH. Open globe injury in Hospital Universiti Sains Malaysia - a 10-year review. Int J Ophthalmol. (2014) 7:486-90. doi: 10.3980/j.issn.2222-3959.2014.03.18

11. Beshay N, Keay L, Dunn H, Kamalden TA, Hoskin AK, Stephanie $\mathrm{L}$ et al. The epidemiology of open globe injuries presenting to a tertiary referral eye hospital in Australia. Injury. (2017) 48:1348-54. doi: 10.1016/j.injury.2017.04.035

12. Court JH, Lu LM, Wang N, McGhee CNJ. Visual and ocular morbidity in severe open-globe injuries presenting to a regional eye centre in New Zealand. Clin Exp Ophthalmol. (2019) 47:469-77. doi: 10.1111/ceo.1 3439

13. Supreeyathitikul P, Chokesuwattanaskul S, Choovuthayakorn J, Patikulsila D, Watanachai N, Kunavisarut P et al. Epidemiology and outcomes following open globe injury in agricultural region, an 11-year experience. Ophthalmic Epidemiol. (2020) 27:246-51. doi: 10.1080/09286586.2020.1716381

14. Kumar DA, Agarwal A, Jacob S, Lamba M, Packialakshmi S, Meduri A. Combined surgical management of capsular and iris deficiency with glued intraocular lens technique. J Refract Surg. (2013) 29:3427. doi: 10.3928/1081597X-20130415-05 
15. Ojuok E, Uppuluri A, Langer PD, Zarbin MA, Thangamathesvaran L, Bhagat N. Predictive factors of enucleation after open globe injuries. Graefes Arch Clin Exp Ophthalmol. (2021) 259:247-55. doi: 10.1007/s00417-02004794-6

16. Savar A, Andreoli MT, Kloek CE, Andreoli CM. Enucleation for open globe injury. Am J Ophthalmol. (2009) 147:595-600 doi: 10.1016/j.ajo.2008.10.017

17. Han YS, Kavoussi SC, Adelman RA. Visual recovery following open globe injury with initial no light perception. Clin Ophthalmol. (2015) 9:1443-48. doi: 10.2147/OPTH.S8 7852

18. Zigiotti GL, Cavarretta S, Morara M, Nam SM, Ranno S, Pichi F, et al. Standard enucleation with aluminium oxide implant (bioceramic) covered with patient's sclera. Sci World J. (2012) 2012:4. doi: 10.1100/2012/48 1584

19. Agrawal R, Wei HS, Teoh S. Predictive factors for final outcome of severely traumatized eyes with no light perception. BMC Ophthalmol. (2012) 12:16. doi: 10.1186/1471-2415-
Conflict of Interest: The authors declare that the research was conducted in the absence of any commercial or financial relationships that could be construed as a potential conflict of interest.

Publisher's Note: All claims expressed in this article are solely those of the authors and do not necessarily represent those of their affiliated organizations, or those of the publisher, the editors and the reviewers. Any product that may be evaluated in this article, or claim that may be made by its manufacturer, is not guaranteed or endorsed by the publisher.

Copyright (c) 2021 Chen, Han, Zhang and Jin. This is an open-access article distributed under the terms of the Creative Commons Attribution License (CC BY). The use, distribution or reproduction in other forums is permitted, provided the original author(s) and the copyright owner(s) are credited and that the original publication in this journal is cited, in accordance with accepted academic practice. No use, distribution or reproduction is permitted which does not comply with these terms. 